\title{
Needle-based optical coherence tomography for the detection of prostate cancer: a visual and quantitative analysis in 20 patients
}

Berrend G. Muller

Rob A. A. van Kollenburg

Abel Swaan

Evita C. H. Zwartkruis

Martin J. Brandt

Leah S. Wilk

Mitra Almasian

A. Wim Schreurs

Dirk J. Faber

L. Rence Rozendaal

Andre N. Vis

Jakko A. Nieuwenhuijzen

Jeroen R. J. A. van Moorselaar

Jean J. M. C. H. de la Rosette

Daniel Martijn de Bruin

Ton G. van Leeuwen 


\title{
Needle-based optical coherence tomography for the detection of prostate cancer: a visual and quantitative analysis in 20 patients
}

\author{
Berrend G. Muller, ${ }^{a, *}$ Rob A. A. van Kollenburg, ${ }^{a}$ Abel Swaan, ${ }^{\text {a,b }}$ Evita C. H. Zwartkruis, ${ }^{c}$ Martin J. Brandt, ${ }^{b}$ \\ Leah S. Wilk, ${ }^{b}$ Mitra Almasian, ${ }^{b}$ A. Wim Schreurs,, ${ }^{d}$ Dirk J. Faber, ${ }^{b}$ L. Rence Rozendaal, ${ }^{c}$ Andre N. Vis, \\ Jakko A. Nieuwenhuijzen, ${ }^{e}$ Jeroen R. J. A. van Moorselaar, ${ }^{e}$ Jean J. M. C. H. de la Rosette, ${ }^{a, f}$ \\ Daniel Martijn de Bruin, ${ }^{a, b}$ and Ton G. van Leeuwen ${ }^{b}$ \\ aUniversity of Amsterdam, Academic Medical Center, Department of Urology, The Netherlands \\ bUniversity of Amsterdam, Academic Medical Center, Department of Biomedical Engineering and Physics, The Netherlands \\ 'VU University Medical Center, Department of Pathology, Amsterdam, The Netherlands \\ dUniversity of Amsterdam, Academic Medical Center, Department of Instrumental Services, The Netherlands \\ eVU University Medical Center, Department of Urology, Amsterdam, The Netherlands \\ 'Istanbul Medipol University, Department of Urology, Istanbul, Turkey
}

\begin{abstract}
Diagnostic accuracy of needle-based optical coherence tomography (OCT) for prostate cancer detection by visual and quantitative analysis is defined. 106 three-dimensional (3-D)-OCT data sets were acquired in 20 prostates after radical prostatectomy and precisely matched with pathology. OCT images were grouped per histological category. Two reviewers performed blind assessments of the OCT images. Sensitivity and specificity for malignancy detection were calculated. Quantitative analyses by automated optical attenuation coefficient calculation were performed. OCT can reliably differentiate between fat, cystic, and regular atrophy and benign glands. The overall sensitivity and specificity for malignancy detection was $79 \%$ and $88 \%$ for reviewer 1 and $88 \%$ and $81 \%$ for reviewer 2. Quantitative analysis for differentiation between stroma and malignancy showed a significant difference $\left(4.6 \mathrm{~mm}^{-1}\right.$ versus $5.0 \mathrm{~mm}^{-1}$ Mann-Whitney $U$-test $\left.p<0.0001\right)$. A Kruskal-Wallis test showed a significant difference in median attenuation coefficient between stroma, inflammation, Gleason 3 , and Gleason 4 (4.6, 4.1, 5.9, and $5.0 \mathrm{~mm}^{-1}$, respectively). However, attenuation coefficient varied per patient and a related-samples Wilcoxon signed-rank test showed no significant difference per patient $(p=0.17)$. This study confirmed the one to one correlation of histopathology and OCT. Precise matching showed that most histological tissues categories in the prostate could be distinguished by their unique pattern in OCT images. In addition, the optical attenuation coefficient can play a role in the differentiation between stroma and malignancy; however, a per patient analysis of the optical attenuation coefficient did not show a significant difference. $\odot$ The Authors. Published by SPIE under a Creative Commons Attribution 3.0 Unported License. Distribution or reproduction of this work in whole or in part requires full attribution of the original publication, including its DOI. [DOI: 10.1117/1.JBO.23.8.086001]
\end{abstract}

Keywords: optical coherence tomography; prostate; needle-based; validation; histopathology; prostate cancer.

Paper 180109R received Feb. 19, 2018; accepted for publication Jul. 18, 2018; published online Aug. 9, 2018.

\section{Introduction}

Prostate cancer consisted $26 \%$ of the new cancer diagnoses in 2015 , and it was the second most common cause of cancerrelated death in the USA. ${ }^{1}$ Prostate specific antigen (PSA) screening led to an increase in the diagnosis of low- and intermediate risk prostate cancer. ${ }^{2}$ Focal therapy is emerging as an attractive treatment option for these patients since it only treats the cancerous part of the prostate while leaving the remaining part unharmed. Focal therapy has significantly fewer side effects when compared to radical treatment. ${ }^{3-9}$ However, accurate imaging on a microscopic level is crucial for case selection and the application of focal therapy.

Imaging technologies that are currently investigated for prostate cancer include multiparametric MRI and transrectal ultrasound (TRUS). ${ }^{10}$ These technologies provide information on a macroscopic level with voxel sizes of $\sim 1 \mathrm{~mm}^{3}$ on MRI and pixel sizes of $\sim 0.2 \mathrm{~mm} \times 0.2 \mathrm{~mm}$ on TRUS. Standard histopathology

*Address all correspondence to: Berrend G. Muller, E-mail: B.G.Muller@amc uva.nl provides diagnostic information on prostate cancer on a microscopic level. This process, however, is time consuming, moderately reproducible between observers, and produces artifacts on subsequent radiological images. ${ }^{11,12}$ An imaging modality that provides reproducible instant information on a microscopic level and that can be used intraoperatively and in the outpatient department would make the process of diagnosis, treatment, and monitoring faster (automated image processing) and more accurate (less open for interpretation error).

Optical coherence tomography (OCT) is a high-resolution imaging method in which contrast is based on differences between light scattering of tissue structures. ${ }^{13}$ Although the imaging depth is limited to $\sim 2 \mathrm{~mm}$, OCT has an important advantage over standard histopathology, because OCT can, either non- or minimally invasively, be applied in vivo, using scan heads or probes in conjunction with balloons, catheters, or needles. Recent developments in needle-based OCT enable minimally invasive puncturing of tissue and therefore in-tissue imaging. ${ }^{4,15}$ Furthermore, the OCT signal can be quantitatively analyzed, thereby providing tissue-specific parameters such as the optical attenuation coefficient. ${ }^{14,16}$ The optical attenuation 
coefficient can function as a measure for tissue density. In previous studies, one to one matching of the OCT images with histology remained challenging, ${ }^{14,16}$ whereas precise histopathology correlation is essential to draw well-founded conclusions from the measured OCT data and to understand which tissue types in the prostate can be distinguished by OCT. Therefore, a customized prostate measurement and slicing device was developed which facilitates the necessary one to one correlation of OCT images of fresh prostate tissue with histology. It has been demonstrated that OCT is able to characterize tissue structures as seen on histopathology. ${ }^{17-19}$ In addition, the tissue density is expected to translate into a different attenuation coefficient when compared with benign tissue. The objectives of the present study are to identify unique structural characteristics in needle-based OCT images, which by pathology are proven benign or malignant prostatic tissues, based on visual parameters and/or quantitative analyses by means of the optical attenuation coefficients. We hypothesize that the obtained structural characteristics and quantitative parameters can be translated into a diagnostic accuracy that approaches biopsy levels. The study is performed according to the IDEAL guidelines for the validation of medical devices ${ }^{20}$ and according to the STARD criteria for diagnostic studies. $^{21}$

\section{Methods}

A prospective observational ex vivo study was performed in 20 prostates immediately after radical prostatectomy. The hospital's ethical board waived the need for evaluation.

\subsection{Participants}

Patients at least 18 years of age, diagnosed with prostate cancer, and scheduled for radical prostatectomy were eligible for inclusion in this study. Potentially eligible patients were identified at the outpatient clinic of the urology department at the VU University Medical Center in Amsterdam between August and November 2014. Inclusion was on a consecutive basis.

\subsubsection{Test methods}

OCT measurements were recorded using a commercially available C7-XR ${ }^{\text {tm }}$ Imaging System interfaced to a C7 dragonfly ${ }^{\mathrm{tm}}$ imaging probe (St. Jude Medical, St. Paul, Minnesota) (Fig. 1). The system uses a wavelength of $1300 \mathrm{~nm}$ with a bandwidth of $55 \mathrm{~nm}$, with a scanrate of 100 frames/s (500 A-lines/frame). ${ }^{22,23}$ The imaging probe was inserted into a transparent intravascular (IV)-needle catheter (Terumo Surflo ${ }^{(} 18 \mathrm{G} \times 2 \frac{1}{2}$ ) allowing tissue puncturing. A detailed description of the device, its adaption for prostate imaging, and the measurement protocol were provided in a previous paper. ${ }^{14}$ Briefly, the rotating fiber-optic probe with an outer diameter of $2.7 \mathrm{Fr}(0.9 \mathrm{~mm})$ produces cross-sectional images with an axial resolution of 10 to $15 \mu \mathrm{m}$ and lateral resolution of 20 to $40 \mu \mathrm{m}$. ${ }^{24}$ A 360-deg probe rotation provides $504 \mathrm{~A}$-scans of $\sim 5 \mathrm{~mm}$. The 504 A-scans were converted into one cross-sectional image or B-scan. In the cylindrical configuration, the B-scan is thus presented with a diameter of $10 \mathrm{~mm}$. The automatic pullback system scans over a trajectory of $54 \mathrm{~mm}$ in $\sim 5.4 \mathrm{~s}$, producing a 540-frame/B-scan dataset, resulting in a cylindrical scan of the prostate tissue of $54 \mathrm{~mm} \times 10 \mathrm{~mm}$, with an imaging depth of $2 \mathrm{~mm}$ limited by scattering. ${ }^{14}$

Ex vivo OCT measurements were performed in fresh tissue at the pathology department directly following radical resection.

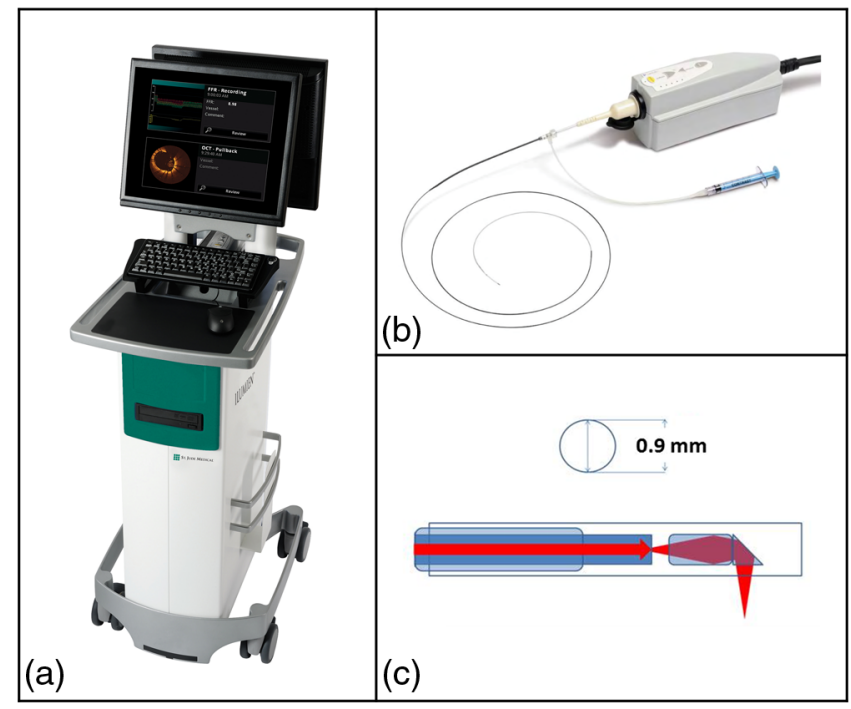

Fig. 1 The St. Jude OCT system. (a) C7-XR ${ }^{\text {tm }}$ OCT console. (b) The C7 dragonfly OCT imaging probe connected to the OCT driver containing the optical fiber and focusing optics and electronic components for measurements. (c) Schematic detail of the OCT probe: the light is deflected perpendicular to the probe's axis into the tissue while the probe rotates and is pulled back, resulting in a cylindrically scanned OCT volume of the tissue surrounding the probe.

Four (in a small prostate) or six (in a large prostate) intravascular (IV)-catheters were placed in the prostate using the customized tool as described in previous work. ${ }^{25}$ After removal of the insertion needle, the C7 dragonfly ${ }^{\mathrm{tm}}$ OCT probe was inserted into the in-situ transparent IV catheter and OCT measurements of the prostate tissue were acquired.

\subsubsection{Histopathological evaluation}

The prostate was sliced after $48 \mathrm{~h}$ formaldehyde fixation. For slicing, the knife was guided through the OCT imaging trajectory (Fig. 2). ${ }^{25}$ Whole mount histopathological slides were produced and stained with hematoxylin and eosin according to the standard VUMC pathology protocol. The histological diagnosis was made by an expert uropathologist who was informed about biopsy and clinically relevant findings but blinded for the OCT results. Afterward, all whole mount slides were digitized. The pathologist performed the marking and annotation of histological structures in the digitized whole mount slides using annotation software designed by our department. Nine histopathological tissue categories were annotated: cystic atrophy, regular atrophy, benign glands, stroma, inflammation, fat and malignancy Gleason pattern 3, Gleason pattern 4, and Gleason pattern 5 .

\subsubsection{Data analysis}

Measurement of the histopathological OCT trajectory length was performed using the pathology annotation software after digital scale calibration to correct for prostate shrinkage after fixation. A starting point was defined at the border of the prostate. Total trajectory length and all distances from the beginning of the trajectory to marked histological structures were measured. This procedure was performed for the histological structures on both sides of the trajectory [ventral (painted red and yellow) and dorsal (painted blue and green)]. The OCT scan 


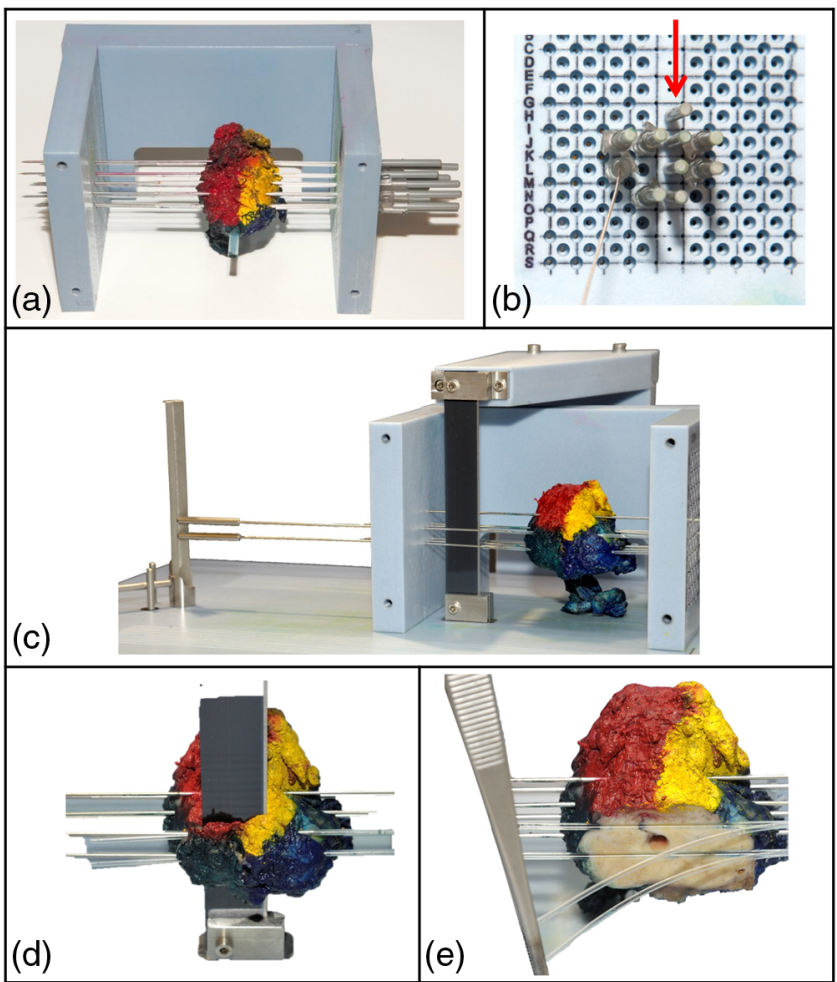

Fig. 2 Customized prostate measurement and slicing tool. (a) First, the prostate is fixed between two grids, ensuring parallel IV catheter insertion for measurements. Four stabilizing needles in the middle ensure that the prostate does not move during slicing (red arrow in b). (b) OCT measurements are performed. Afterward, the prostate is fixated for $48 \mathrm{~h}$ in formalin. (c) The prostate is placed in the slicing device, with knife guiders surrounding the knife, ensuring that the prostate is sliced perfectly through the OCT imaging trajectory. (d and e) The procedure is extensively described in earlier work. ${ }^{25}$

creates a 360-deg view of the tissue. Histopathology was twodimensional (2-D) and thus contained only information in one plane. Rotational matching was ensured by overlapping identical structures seen in histopathology and OCT, as is indicated by the arrows in Fig. 3. The urethra and outer shape of the prostate provided a rough estimation of orientation. Cysts were used for final precise orientation.

The B-scan locations were matched to the distances measured in the histological slides. Based on this information, a virtual overlay of the OCT scan over the histology was made. In this way, the histological classification of every single B-scan was deduced.

\subsubsection{Qualitative data analysis}

For each of the nine histological categories, locations were selected where opposing sites (ventral and dorsal to the imaging trajectory) were classified in the same category; we refer to these regions as the regions of interest (ROIs). On these locations, we assume that all tissue surrounding the catheter channel has the same histological characteristics. The corresponding OCT Bscans were saved to a database. Subsequently, 110 B-scans across the histological categories were selected by RK, randomized, and offered to two reviewers (BM and AS) blinded for the biopsy histology assessment and further clinical information. Prior to analysis, the two reviewers received a short training based on example images and a short qualitative description

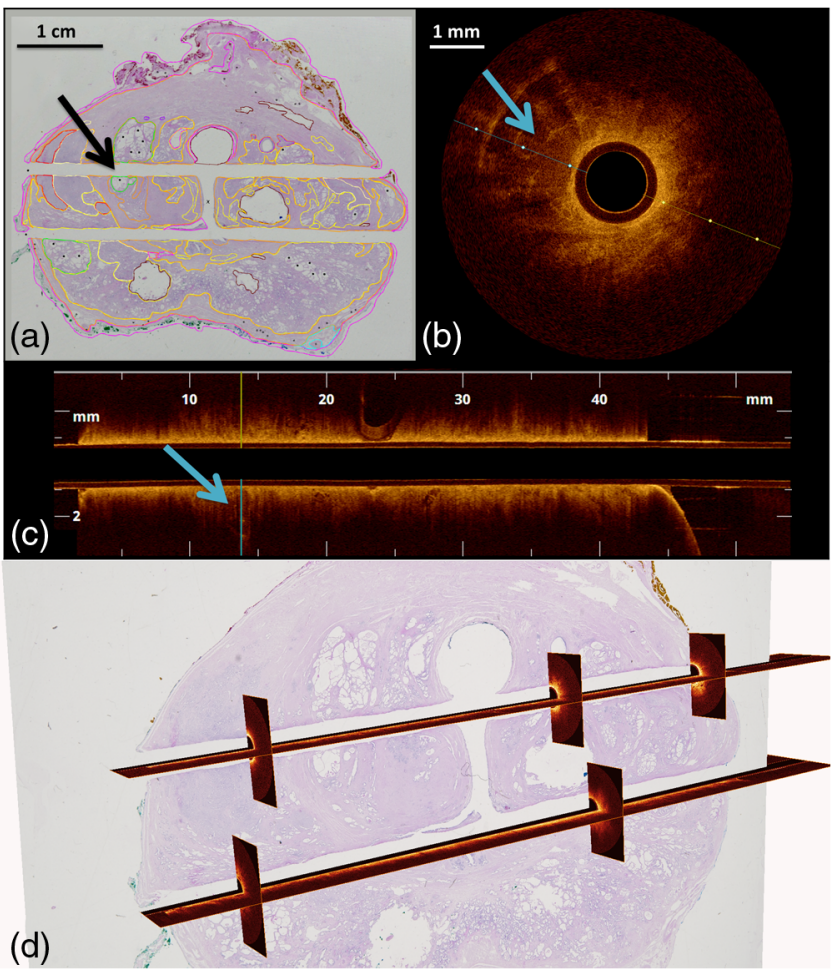

Fig. 3 Correlation of histology and OCT. (a) Digitized H\&E-stained whole mount slide of the prostate. The two (horizontal) trajectories from the OCT measurements are clearly visible. The pathologist annotates the tissue types in different colors. The black arrow indicates an atrophic cyst. (b) An OCT B-scan at the level of the atrophic cyst from (a). The blue arrow shows the same atrophic cyst. The blueyellow line corresponds with the longitudinal cross section of the cylindrical dataset depicted in (c). (c) A longitudinal cross section of the OCT scan, also used for final rotational correlation with histology. The blue arrow indicates the atrophic cyst, seen in (a) and (b). (d) 3-D visualization of OCT images matched one-to-one with histopathology.

of the OCT images. Both reviewers independently assigned the B-scans to one of the nine histological categories for comparison to the histological classification.

\subsubsection{Quantitative data analysis}

Quantification of the optical attenuation coefficient was performed using custom-written code (Matlab 7.11.0 R2010b, The Mathworks Inc., Natick, Massachusetts). The optical attenuation coefficients from the ROIs were obtained by processing the data according to steps shown in Fig. 4. The region of interest (ROI) is divided into fit regions (FR) with the following dimensions: $(31 \times 6$ A-lines $)$. Each separate A-line in an FR was laterally averaged, and attenuation coefficient $\left(\mu_{\mathrm{OCT}}\right)$ was fitted according to the method in Appendix yielding $1 \mu_{\mathrm{OCT}}$ per fit region, which correspond to a specific location in the histopathology. These values were used for analysis.

The process is described in detail in Appendix and shown in Fig. 5. We used our experience from previous work to design the program. ${ }^{14,16,26}$

In order to improve the data quality, a margin of error was included for the OCT-histopathology correlation process. Five B-scans, covering $0.5 \mathrm{~mm}$ in scan length, at the beginning and end of each ROI were deleted. The remaining ROIs had to 


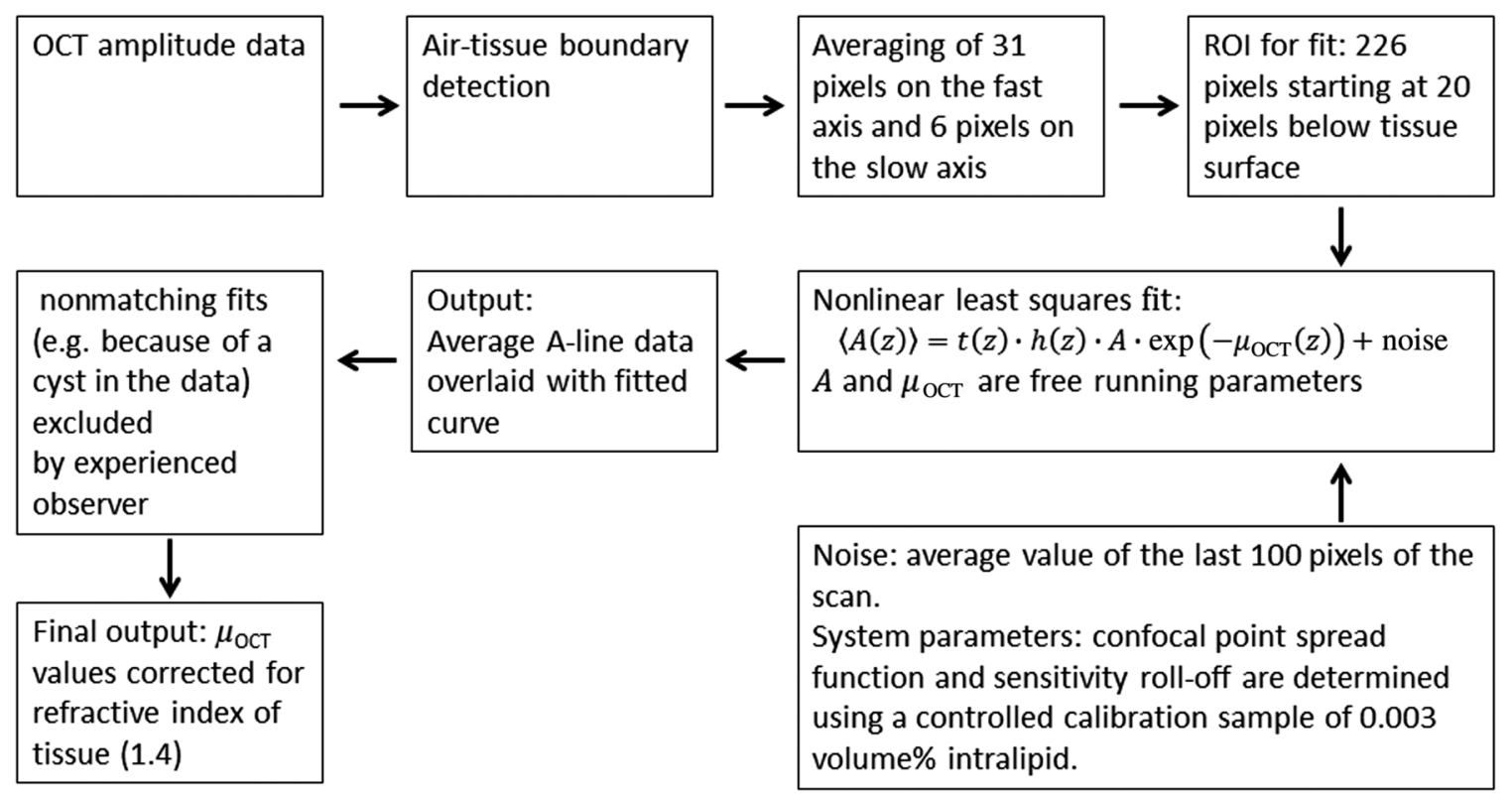

Fig. 4 Automated quantitative analysis. The process is described in detail in Appendix.

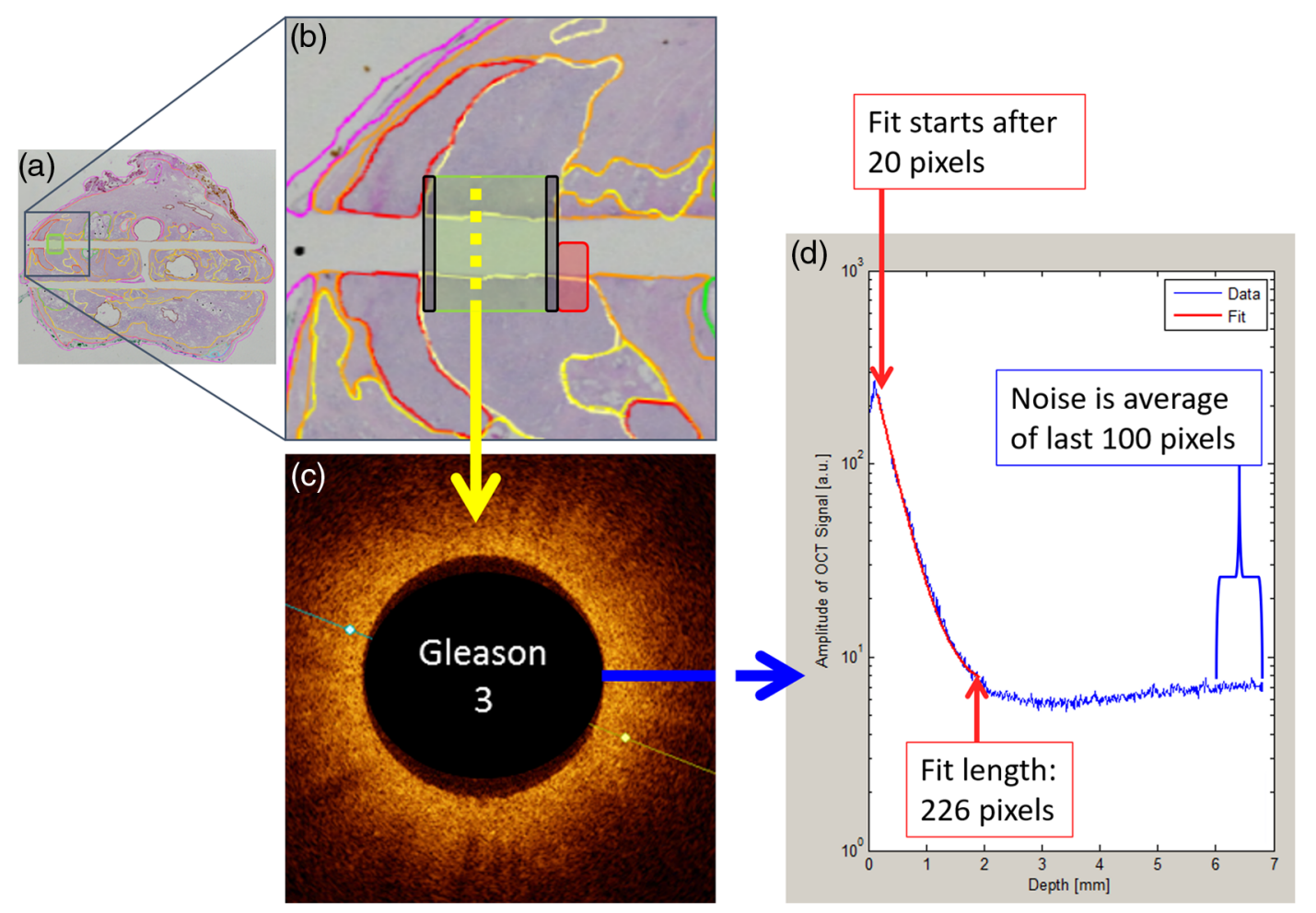

Fig. 5 (a) First, tissue types were assigned by the pathologist, (b) Regions were selected with the same histopathology on both sides of the imaging trajectory, 5 B-scans on both sides of the ROI were taken as a safety margin and not included in the analysis. The B-scans in this region (dotted yellow line and example in c) were further analyzed as described in Appendix (d). The fit starts 20 pixels after reaching tissue surface, the fitlength is 226 pixels. An average of the final 100 pixels was used as a measurement of the noise.

contain at least six B-scans for final inclusion. The attenuation coefficients of these regions were stored in a database based on the histologic classification. The determined values for stroma, inflammation, and all malignancy categories were grouped per patient. In case of missing data of whole mount slides or OCT scans, the specific OCT scan/histology combination was excluded from the analysis.

\subsubsection{Statistics}

The results of the visually reviewed OCT B-scan were compared to the whole mount histopathology slides. In addition, answers were grouped based on similar histological classification. Finally, all scores were grouped for benign and malignant histology. Sensitivity, specificity, false negative, and false positive 
calculations were performed manually using a $2 \times 2$ table. The interobserver agreement expressed in kappa was calculated for every category. Kappa $(k)<0$ was defined as "poor agreement," 0 to 0.20 "slight agreement," 0.21 to 0.4 "fair agreement," 0.41 to 0.60 "moderate agreement," 0.61 to 0.8 "substantial agreement," and above 0.81 "almost perfect agreement."

Optical attenuation coefficients of malignancy Gleason patterns 3, 4, and 5 were grouped. With a Kolmogorov-Smirnov test, data were tested for a normal distribution. A MannWhitney $\mathrm{U}$ test was performed for comparison of the attenuation coefficient of stroma and malignancy, using MedCalc v 15.8. A Kruskal Wallis test was performed to compare attenuation coefficients of stroma, inflammation, Gleason 3, and Gleason 4 individually. A $p$-value of $\leq 0.05$ stated significance. In addition, receiver operating characteristic (ROC) curve analyses were performed. The area under the curve (AUC) was calculated to determine test accuracy.

\section{Results}

\subsection{Patient Characteristics}

Patient characteristics are described in Table 1: 20 consecutive patients were included. On these 20 prostates, 106 three-dimensional (3-D)-OCT measurements were performed. The mean age was 65 years with a mean PSA of $11.5 \mathrm{ng} / \mathrm{ml}$ at the time of surgery. All patients except one underwent prostate biopsies with Gleason scores ranging from $3+3$ to $4+4$. One patient was included based on benign prostate hyperplasia transurethral resection results. Histologic specimen examination provided Gleason scores ranging from $3+4$ to $4+5$ and one benign specimen. Seminal vesicle invasion, extracapsular invasion, pelvic lymph node involvement, and positive resection margins were present in two, six, one, and five patients, respectively.

\subsection{OCT and Histology Correlation}

A total number of 52 whole mount histopathological slides were available. The OCT trajectories were visible in 50 slides. Malignancy was visible in 19 histology slides in 13 prostates. Twenty-one slides were damaged during production, resulting in areas with data loss. Matching of OCT scans with histology was achieved with high precision in 36 slides. The urethra and outer boundaries of the prostate contributed to the first rotational orientation. Mainly cysts but also other landmarks such as the urethra provided data for precise rotational orientation as can be seen in Fig. 3. Rotational inaccuracy was estimated at $10 \mathrm{deg}$.

\subsection{Qualitative Description of B-Scans}

The earlier selected representative OCT B-scans that were grouped per histopathological tissue type were reviewed for identification of unique marks on OCT. Figure 6 shows a representative B-scan per category. Review of the B-scans showed that part of the histopathology was well identifiable by OCT based on unique characteristics. Cystic atrophy [Fig. 6(1)] was visually identified by cavities $(>0.5 \mathrm{~mm})$, divided by septae. The content of the cavities appears opaque due to backscattered light. Regular atrophy [Fig. 6(2)] has smaller (0.1 to $0.3 \mathrm{~mm}$ ), dark, more grouped cavities than those found in cystic atrophy. Benign glands [Fig. 6(3)] have even smaller, mostly grouped cavities $(\leq 0.1 \mathrm{~mm})$. The cavities could be dark or opaque. Fat [Fig. 6(4)] had a honeycomb structure, consisting of a unique pattern of bright stripes, alternated by dark dots.
Table 1 Patient characteristics.

\begin{tabular}{|c|c|c|}
\hline Patients & & $n=20$ \\
\hline Age at operation (years) & & $\begin{array}{c}\text { mean: } 64.5 \\
\text { (range: } 55 \text { to } 76 \text { ) }\end{array}$ \\
\hline PSA at operation $(\mathrm{ng} / \mathrm{ml})$ & & $\begin{array}{c}\text { mean: } 11.5 \\
\text { (range: } 2.9 \text { to } 36)^{2}\end{array}$ \\
\hline Biopsies (Gleason) & $3+3$ & $n=5$ \\
\hline & $3+4$ & $n=11$ \\
\hline & $4+3$ & $n=2$ \\
\hline & $4+4$ & $n=1$ \\
\hline Total number of cores & & $\begin{array}{l}\text { mean: } 9.6 \\
\text { (range: } 6 \text { to } 15 \text { ) }\end{array}$ \\
\hline Number of cores positive & & $\begin{array}{c}\text { mean: } 4 \\
\text { (range: } 1 \text { to } 7)^{\mathrm{b}}\end{array}$ \\
\hline Specimen (Gleason) & $0+0$ & $n=1$ \\
\hline & $3+4$ & $n=7$ \\
\hline & $4+3$ & $n=11$ \\
\hline & $4+5$ & $n=1$ \\
\hline Seminal vesicle invasion & & $n=2$ \\
\hline Extracapsular invasion & & $n=6$ \\
\hline Pelvic lymph node dissection & & $n=7$ \\
\hline Lymph node involvement & & $n=1$ \\
\hline Resection margins positive & & $n=5$ \\
\hline
\end{tabular}

a2.9 under Combodart.

b One patient underwent a radical prostatectomy based on a histopathological Gleason score $4+4$ after transurethral resection of the prostate.

The remaining histopathological classifications were more difficult to distinguish by OCT. Malignancy Gleason patterns 3 and 4 [Figs. 6(5) and 6(6)] present as homogeneous tissue structures. High signal surrounding the probe and low signal in depth represented a low signal penetration $(<1 \mathrm{~mm})$, probably due to a high cell density. Stroma [Fig. 6(7)], similarly to malignant tissue, appeared homogeneous but with an increased signal depth ( $\geq 1 \mathrm{~mm}$ ). Parallel ellipsoidal-shaped lines on both sides of the probe were unique for stroma and probably caused by fibrotic tissue, yet this was not seen in all stromal B-scans. Inflammation [Fig. 6(8)] had a homogeneous pattern with high cell density and signal penetration of $\sim 1 \mathrm{~mm}$, with vague outer boundaries.

\subsection{Qualitative Blind Assessment of B-Scans}

Blind assessment of OCT B-scans was done by two reviewers (see Table 2). The percentages represent the number of B-scans correctly scored by the reviewer. Grouping scores for categories with similar histology and intended treatment improved the results. Combining benign glands, cystic atrophy, and regular atrophy resulted in scores of $100 \%$ and $88.9 \%$. The combination 


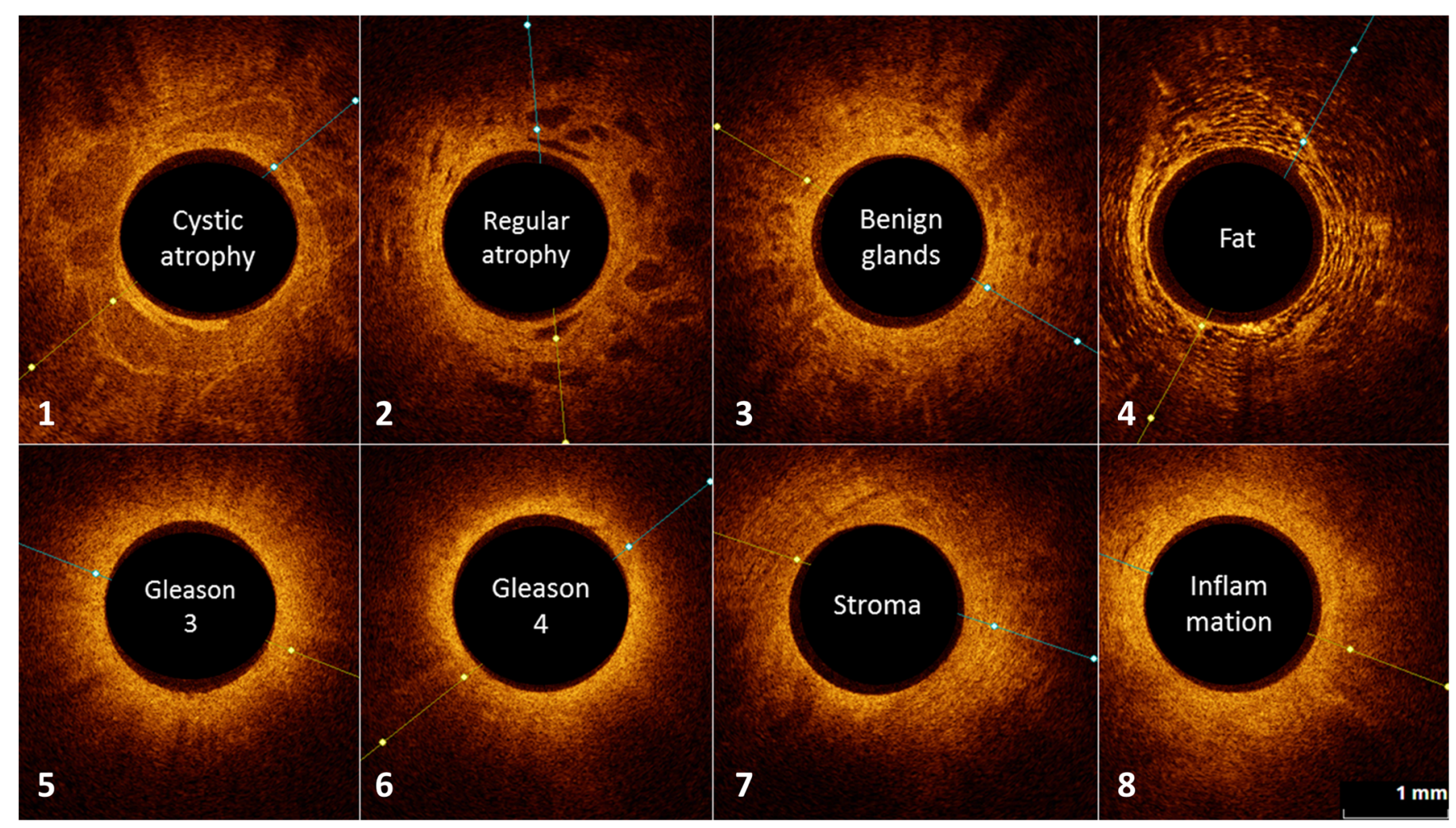

Fig. 6 Histological tissue characteristics seen in OCT. The inner part of the OCT probe and the IV catheter in the middle are made black.

of stroma and inflammation resulted in scores of $56 \%$ and $59 \%$. Malignancy Gleason patterns 3, 4, and 5 resulted in correct scores of $79 \%$ and $88 \%$. In particular, stroma and inflammation were most often misidentified, totaling $n=17$ and $n=17$ out of 41 for reviewers 1 and 2, respectively. Grouping malignant and benign scores gave an overall sensitivity and specificity for malignancy detection of $79 \%$ and $88 \%$ for reviewer 1 , and $88 \%$ and $81 \%$ for reviewer 2 . Negative predictive values were high (94\% and $96 \%$ for reviewers 1 and 2, respectively). Positive predictive values were relatively low (66\% and 57\% for reviewers 1 and 2, respectively).

The interobserver agreement on OCT images was calculated and resulted in moderate agreement between observers (weighted kappa of 0.50 for overall test values).

When test results were combined into similar groups with similar histology and intended treatment, benign cystic structures (benign/cystic and regular glands), benign stromal structures (stroma/inflammation), and malignant structures (Gleason pattern 3/4/5), there was substantial agreement between observers (kappa of 0.64). When the results were grouped in two groups: benign structures (stroma and inflammation) and malignant structures (Gleason pattern 3/4/5), there was moderate agreement between observers (kappa of 0.57 ).

\subsection{Quantitative Analysis: Attenuation Coefficient}

Automated attenuation coefficient calculations were performed for additional differentiation between stroma and malignancy. Twelve patients were included; eight patients could not be included in the analysis, as the tumor or inflammation was not scanned by OCT.

We noticed that the attenuation coefficient (malignant and benign) differed per patient.
Table 2 Results of visual OCT B-scan scoring.

\begin{tabular}{|c|c|c|c|c|}
\hline \multirow[b]{2}{*}{ Scores } & \multicolumn{2}{|c|}{ Reviewer 1} & \multicolumn{2}{|c|}{ Reviewer 2} \\
\hline & Exact & Percentage & Exact & Percentage \\
\hline Benign glands & $15 / 16$ & $94 \%$ & $11 / 16$ & $69 \%$ \\
\hline Cystic atrophy & $8 / 9$ & $89 \%$ & $7 / 9$ & $78 \%$ \\
\hline Regular atrophy & $8 / 11$ & $72 \%$ & $4 / 11$ & $36 \%$ \\
\hline Stroma & $16 / 31$ & $52 \%$ & $13 / 31$ & $42 \%$ \\
\hline Gleason pattern 3 & $2 / 5$ & $40 \%$ & $3 / 5$ & $60 \%$ \\
\hline Gleason pattern 4 & $9 / 19$ & $47 \%$ & $6 / 19$ & $32 \%$ \\
\hline Inflammation & $1 / 10$ & $10 \%$ & $4 / 10$ & $40 \%$ \\
\hline Fat & $9 / 9$ & $100 \%$ & $9 / 9$ & $100 \%$ \\
\hline \multicolumn{5}{|l|}{ Grouped scores } \\
\hline Benign, cystic, regular & $36 / 36$ & $100 \%$ & $32 / 36$ & $89 \%$ \\
\hline Stroma, inflammation & $23 / 41$ & $56 \%$ & $24 / 41$ & $59 \%$ \\
\hline Malignancy $3,4,5$ & $19 / 24$ & $79 \%$ & $21 / 24$ & $88 \%$ \\
\hline \multicolumn{5}{|c|}{ Malignancy detection (GI 3/4/5) } \\
\hline Sensitivity & & $79 \%$ & & $88 \%$ \\
\hline Specificity & & $88 \%$ & & $81 \%$ \\
\hline NPV & & $93 \%$ & & $96 \%$ \\
\hline PPV & & $65 \%$ & & $57 \%$ \\
\hline
\end{tabular}


The attenuation coefficient was higher for malignancy than for stroma $\left(5.0 \mathrm{~mm}^{-1}\right.$ versus $\left.4.6 \mathrm{~mm}^{-1}\right)$. This yields for most patients except four (Table 3, Fig. 7). Data were tested for a normal distribution with a Kolmogorov-Smirnov test, and normality was rejected in both benign and malignant data $(p<0.0001)$.
A Mann-Whitney $U$ test showed a significant difference in attenuation coefficient $(p<0.0001)$. Additional analyses with an ROC curve as accuracy measurement of the attenuation coefficient in discrimination of malignancy and stroma showed an AUC of 0.62. For a threshold value of $4.6 \mathrm{~mm}^{-1}$, the test has

Table 3 Overview of the mean attenuation $\left(\mathrm{mm}^{-1}\right)$ coefficient per histological category per patient. Only patients with malignant OCT data were included in the analysis. (standard deviation) [number of b-scan excluded/total number of b-scans (percentage of b-scans excluded)].

\begin{tabular}{|c|c|c|c|c|}
\hline & AC stroma $\left(\mathrm{mm}^{-1}\right)$ & AC inflammation $\left(\mathrm{mm}^{-1}\right)$ & AC Gleason $3\left(\mathrm{~mm}^{-1}\right)$ & AC Gleason $4\left(\mathrm{~mm}^{-1}\right)$ \\
\hline \multirow[t]{2}{*}{1} & $4.9(1.0)$ & $4.5(0.8)$ & - & $6.5(1.1)$ \\
\hline & {$[30 / 95(32 \%)]$} & {$[26 / 80(33 \%)]$} & & [1/32 (3\%)] \\
\hline 2 & $\begin{array}{c}4.5(0.8) \\
{[256 / 672(38 \%)]}\end{array}$ & - & $\begin{array}{c}4.8(0.8) \\
{[35 / 80(44 \%)]}\end{array}$ & - \\
\hline \multirow[t]{2}{*}{3} & $5.00(0.9)$ & $4.8(0.6)$ & - & $4.9(0.7)$ \\
\hline & {$[307 / 672(46 \%)]$} & {$[13 / 32(41 \%)]$} & & {$[184 / 1136(16 \%)]$} \\
\hline 4 & - & - & - & - \\
\hline 5 & - & - & - & - \\
\hline \multirow[t]{2}{*}{6} & $4.6(1.5)$ & - & - & $6.1(1.4)$ \\
\hline & {$[57 / 128(45 \%)]$} & & & [2/48 (4\%)] \\
\hline \multirow[t]{2}{*}{7} & $4.6(0.9)$ & - & - & $4.6(0.6)$ \\
\hline & {$[78 / 208(38 \%)]$} & & & [89/384 (23\%)] \\
\hline \multirow[t]{2}{*}{8} & $4.5(0.9)$ & $4.5(1.4)$ & - & $5.6(1.0)$ \\
\hline & [256/672 (38\%)] & {$[8 / 16(50 \%)]$} & & {$[2 / 16(13 \%)]$} \\
\hline 9 & - & - & - & - \\
\hline \multirow[t]{2}{*}{10} & $4.8(0.8)$ & - & - & $4.6(0.6)$ \\
\hline & [98/400 (25\%)] & & & {$[15 / 48(31 \%)]$} \\
\hline \multirow[t]{2}{*}{11} & $4.8(1.1)$ & - & - & $5.2(0.8)$ \\
\hline & {$[55 / 96(57 \%)]$} & & & [37/128 (29\%)] \\
\hline 12 & - & - & - & - \\
\hline \multirow[t]{2}{*}{13} & $4.3(0.8)$ & - & $6.1(0.7)$ & $6.1(0.4)$ \\
\hline & {$[93 / 144(65 \%)]$} & & {$[1 / 48(2 \%)]$} & {$[4 / 16(25 \%)]$} \\
\hline 14 & - & - & - & - \\
\hline 15 & - & - & - & - \\
\hline 16 & - & - & - & - \\
\hline \multirow[t]{2}{*}{17} & $4.6(0.7)$ & - & - & $4.9(0.9)$ \\
\hline & {$[48 / 112(43 \%)]$} & & & [182/640 (28\%)] \\
\hline \multirow[t]{2}{*}{18} & $6.4(2.1)$ & - & - & $5.4(1.0)$ \\
\hline & {$[61 / 128(48 \%)]$} & & & $[688 / 2240)(31 \%)]$ \\
\hline 19 & - & - & - & - \\
\hline \multirow[t]{2}{*}{20} & $4.4(0.8)$ & - & - & $4.3(0.8)$ \\
\hline & {$[39 / 80(49 \%)]$} & & & {$[25 / 48(52 \%)]$} \\
\hline Mean & $4.4(0.9)$ & $4.7(1.0)$ & $5.5(1.0)$ & $5.1(0.9)$ \\
\hline
\end{tabular}


a sensitivity of $68 \%$ and a specificity of $49 \%$ (Fig. 8). Separate categories were analyzed with a Kruskal Wallis test (Fig. 9), which showed a significant difference in median attenuation coefficient between stroma, inflammation, Gleason 3, and Gleason 4 (4.6, 4.1, 5.9, and $5.0 \mathrm{~mm}^{-1}$, respectively) $(p<0.05)$. Since attenuation coefficient strongly differed per patient, we also decided to perform a paired samples Wilcoxon signedrank test. The dot-and-line diagram is shown in Fig. 10. The paired samples $T$-test does not show a significant difference in optical attenuation coefficient between benign and malignant tissues in the prostate per individual patient $p=0.17$.

\section{Discussion}

This study demonstrates that needle-based OCT can identify unique tissue patterns in benign and malignant prostatic tissue and is even able to distinguish benign tissue from malignant prostate $(p<0.05)$. Visually, all types of prostatic tissue were identifiable on OCT, although malignancy, stroma, and inflammation present as similar patterns and therefore it was more challenging to differentiate between those categories. Additional computed OCT analysis by means of the attenuation coefficient did contribute to discrimination of benign from malignant tissue in the prostate. However, when analyzed on

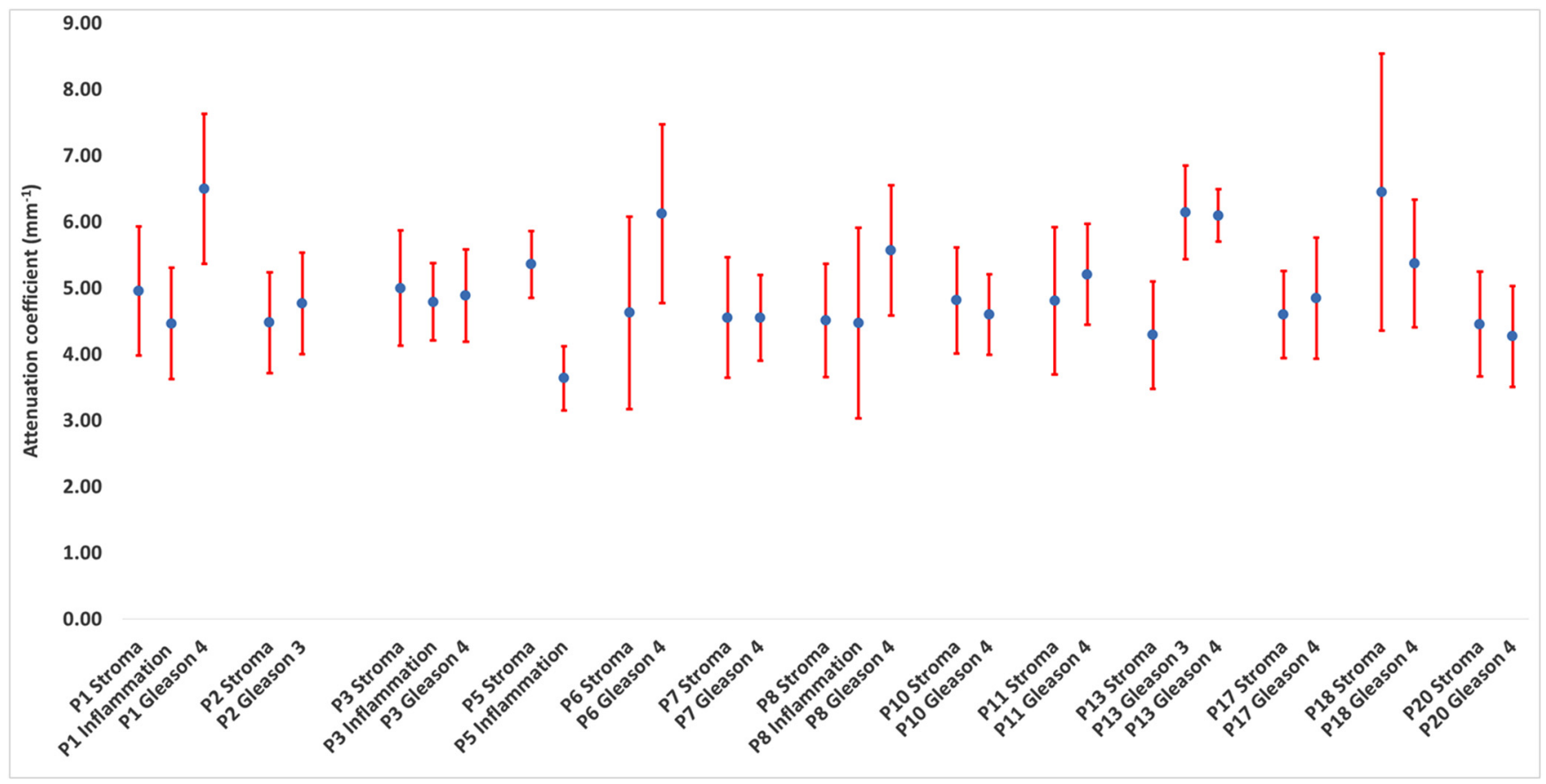

Fig. 7 Per patient analysis of optical attenuation coefficients of histological categories per patient, means with 1 SD error bars.

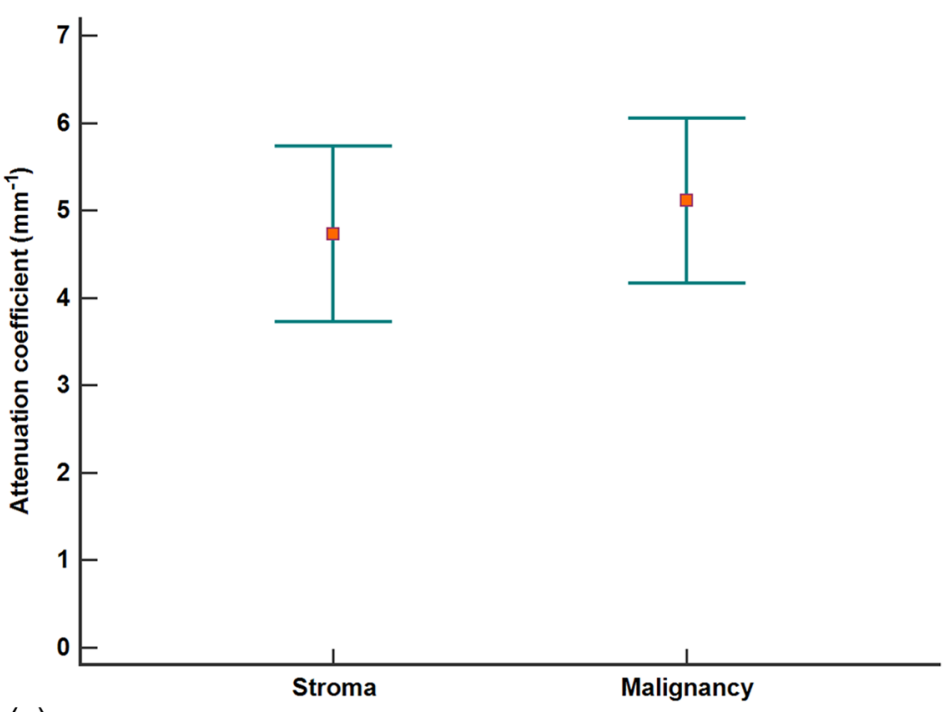

(a)

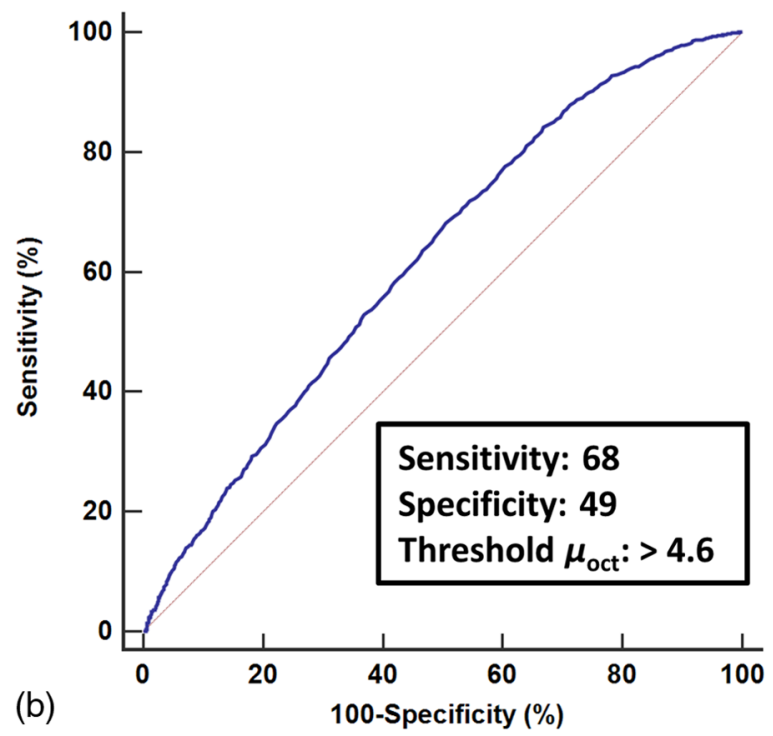

(b)

Fig. 8 (a) Mean attenuation coefficients for stroma and malignancy with 1 SD error bars. (b) ROC analysis. 


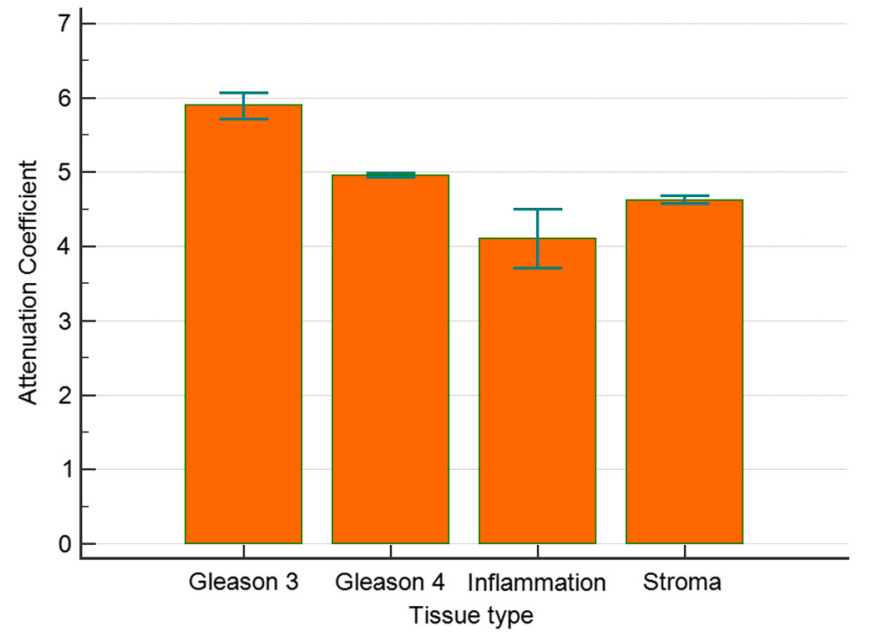

Fig. 9 Bar graph median attenuation coefficients for Gleason 3, Gleason 4, stroma, and inflammation with $95 \%$ confidence intervals for medians.

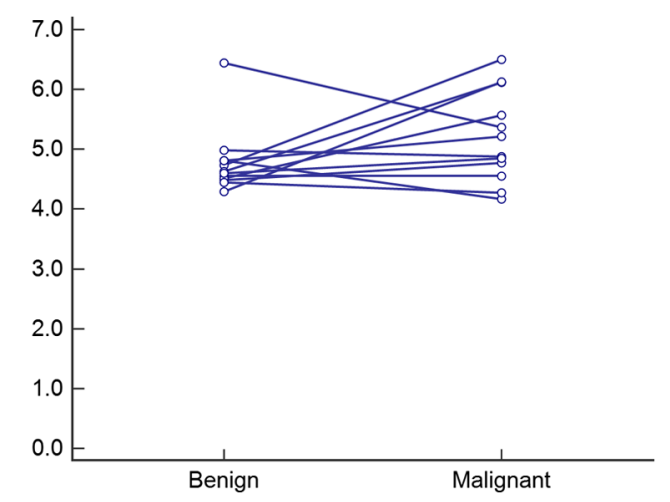

Fig. 10 Dot and line diagram of optical attenuation coefficient analyzed per patient. A Wilcoxon signed-rank test did not show a significant difference between optical attenuation coefficients per patient $p=0.17$.

a per patient basis, there was no significant difference in optical attenuation coefficient. The unique aspect of this study is the one to one correlation of OCT and histopathology in prostate cancer. In previous work, the correlation of histopathology and OCT scans was a serious drawback. ${ }^{10,14}$ For this reason, a customized tool for OCT measurement and prostate slicing was developed. The feasibility of precise correlation of histopathology and OCT of the device was described in earlier work. ${ }^{25}$ Using this method, we performed a single blind qualitative and quantitative accuracy study of OCT in a larger cohort of 20 patients ex vivo.

\subsection{Limitations on Data Matching}

The correlation of 3-D OCT scans and 2-D histopathology was based on two assumptions. First, OCT probe rotation in the prostate tissue was approximated by corresponding structures seen in OCT and histology. The estimated rotational inaccuracy is $10 \mathrm{deg}$, which we consider a small inaccuracy. Second, when both sides of the trajectory contained identical histological structures, it was assumed that all tissue surrounding the OCT probe was from the same histological category. Although this seems plausible, histopathology showed a large heterogeneity in prostatic tissue; therefore, it might be possible that some areas contain tissue from another category. In order to minimize measurement errors, we identified five B-scans on both sides of the ROIs as the margin of error and excluded them from data analysis.

Because the OCT-probe only samples part of the tissue, we missed the tumor in eight patients. For this reason, we did not include this OCT data in the visual assessment and we were not able to analyze the attenuation coefficient on malignancy in these patients.

\subsection{Limitations in Visual Assessment}

For visual assessment, the observers were affiliated with OCT but were not formally trained for detection of prostate cancer. Therefore, it is plausible that their assessment scores can be improved by OCT assessment training. This training could consist of an explanation of the unique tissue characteristics followed by an extended test of prostatic B-scans. This method has been successfully executed before in other imaging studies, e.g., using MRI to visualize prostate cancer, training significantly increased diagnostic accuracy. ${ }^{27,28}$

\subsection{Limitations in Quantitative Assessment Using the Optical Attenuation Coefficient}

It was remarkable that the optical attenuation coefficient for one category (e.g., Gleason 4 ) could differ within a patient with $\mu_{\mathrm{OCT}}$ values ranging 1 to $2 \mathrm{~mm}^{-1}$. This intrapatient variation might be because prostate tissue is heterogonous and is therefore challenging for the pathologist to delineate an area as benign or malignant. Moreover, the pathologist analyzes the whole mount prostatic histology slide using a $40 \times$ microscope and delineates this using software on a digital lower resolution image of the same slide, a process prone to errors. We partially corrected for this by removing $5 \mathrm{~B}$ scans from each side of each ROI as described earlier. Yet, it is also known from histological studies that healthy prostate tissue differs between patients and therefore tumor tissue might differ between patients as well. ${ }^{29}$ Furthermore, a fair amount of fits $(\sim 30 \%)$ was excluded from the analysis because the calculated fit did not exactly match the measured data. Most likely, these specific regions of the prostate are too heterogeneous for a correct fit, e.g., cysts, but they might still have a clinical significance. Technology such as automated texture analysis and pattern recognition could contribute to solve this problem and could increase the performance of OCT for prostate cancer detection. ${ }^{30}$ Furthermore, when more data are available, convolutional neural networks could be trained to perform the analyses on the OCT data, as it now starting in conventional histopathology. ${ }^{31}$

\subsection{Perspective to Other Work in the Field}

Ex vivo application of OCT on the human prostate was first described in the year 2000. 2-D cross sectional OCT scans were generated of specimens after radical prostatectomy. ${ }^{32}$ It was claimed that OCT could distinguish malignant from benign prostate tissue on the basis of architectural differences in the tissue. OCT has also been described for prostatic nerve identification in order to spare them during radical prostatectomy experiments. ${ }^{33,34}$ Dangle et al. ${ }^{35}$ evaluated surgical margins, seminal vesicle invasion, and capsular invasion with OCT ex vivo in prostates after radical prostatectomy. They found that OCT overestimated the amount of margin involvement, but the 
NPV was high. For surgical margins on OCT sensitivity, specificity, PPV, and NPV was 70\%, 84\%, 33\%, and 96\%, respectively. Recently, a study by Lopater et al. ${ }^{36}$ described the initial application of full-field OCT for the detection of prostate cancer in prostate biopsies. This ex vivo OCT imaging method approaches the resolution of traditional histological slides with a resolution of about $1 \mu \mathrm{m}$. The images were scored solely by architectural structures and solely in the 2-D setting, sensitivity, specificity, PPV, and NPV values were $63 \%, 74 \%, 55.5 \%$, and $80 \%$ respectively. In our study, sensitivity $(79 \%$ and $88 \%)$ and specificity ( $88 \%$ and $81 \%$ ) for malignancy detection were higher than in these previous studies. PPVs (66\% and 57\%) and NPVs (94\% and $96 \%$ ) were higher or comparable to these previous studies. Although PPVs were higher than previous studies, they were still considerably lower than the NPVs. This indicates that OCT, as it is investigated here, can be a good test to exclude disease in the prostate. The advantage of the St. Jude C7-XR ${ }^{\mathrm{tm}}$ Intravascular Imaging System is that it is commercially available and applicable in a sterile environment. Consequently, the application of this OCT system in the outpatient clinic or even in a surgery setting is relatively easy. The small probe diameter even allows for in vivo insertion in the prostate. Images are acquired in 3-D, which increases the amount of information substantially when compared to 2-D histopathology. Needle-based OCT lacks the spatial resolution to function as a stand-alone diagnostic modality in prostate cancer. However, it can make the process of conventional histopathology faster and possibly more accurate. Since OCT results are quantifiable, it can reduce interrater variability, which is high for conventional histopathology. ${ }^{37}$

\section{Conclusion}

This study validates one to one correlation of histopathology and OCT using the uniquely designed prostate slicing device in a cohort of 20 patients. Correlation showed that most histological tissues have a unique pattern and therefore could be visually identified on OCT, such as cysts, lines, view in depth, or signal intensity. The abilities of OCT for prostate cancer identification were explored and qualitative visual analysis confirmed the hypothesized high cell density of malignant tissue. In addition, the optical attenuation coefficient contributes to the differentiation between stroma and malignancy, although a per-patient analysis did not show a significant difference. These findings may serve as a basis for an in vivo study combining OCT and prostate biopsy histopathology; allowing comparison of both tests and exploring the clinical potential of OCT in digital pathology of the prostate.

\section{Appendix: Fitting the Optical Attenuation Coefficient}

Quantification of the optical attenuation coefficient was performed using custom-written code (Matlab 7.11.0 R2010b, The Mathworks Inc., Natick, Massachusetts). Methods were derived from our previous work. ${ }^{14,16,26}$ First, OCT amplitude data were loaded into the software. Isolation of tissue-related data from the original data set was achieved by a succession of image processing steps applied to all B-Scans. First, low SNR regions were excluded by applying a pixel value threshold on the amplitude data. Next, to remove the catheter from the B-Scans, an algorithm utilizing region connectivity as well as a priori knowledge about the catheter-tissue geometry was implemented. Following this, a $5 \times 5$ Gaussian low pass filter was applied to the catheter-free images; this step served to improve performance of the ensuing edge detection using the Sobel method. The detected tissue edge was then smoothed further by carrying out local regression using weighted linear leastsquares (assigning lower weight to outliers and zero weight to data outside six mean absolute deviations) and a second-degree polynomial model. OCT amplitude data of the prostate tissue were isolated from the original B-Scan by selecting all data below this smoothed edge. Finally, these data were straightened and then divided into FRs with the following dimensions: $31 \times 6 \times 226$ pixels in the fast axis, slow axis, and depth (located 20 pixels below the tissue edge), respectively. These individual FRs were then laterally averaged, yielding a single average A-line for each FR. Subsequently, an FR-specific attenuation coefficient $\left(\mu_{\mathrm{OCT}}\right)$ was determined by nonlinear least squares fitting the following equation to this average A-line: $\quad A(z)=t(z) \cdot h(z) \cdot A \cdot \exp \left[-\mu_{\mathrm{OCT}}\left(z-z_{0}\right)\right]+$ noise. $A(z)$ is the averaged OCT amplitude in depth, $z$ is the position in depth, $z_{0}$ is the position of the tissue boundary, $A$ and $\mu_{\mathrm{OCT}}$ are free running parameters (amplitude and attenuation coefficient, respectively). The fit always starts 20 pixels below the tissue surface to ensure the absence of tissue edge reflection. The noise is defined as the average of the last 100 pixels of the data. The system dependent parameter $t(z)$ (describing the confocal point spread function) is defined as: $t(z)=\frac{1}{\sqrt{\left(\frac{z-z_{f}}{2 n Z_{R 0}}\right)^{2}+1}}$, where $z_{f}$ is the position of the focus in depth, $Z_{R 0}$ is the Rayleigh length, and $n$ is the refractive index of the medium. The sensitivity roll-off $h(z)$ is defined as: $h(z)=\operatorname{sinc}\left(\frac{\pi}{2} \cdot \frac{z}{z_{\max }}\right) \cdot \exp \left[-\frac{\pi^{2} \cdot s^{2}}{16 \cdot \ln (2)} \cdot\left(\frac{z}{z_{\max }}\right)^{2}\right]$. Here, $z_{\max }$ is the maximal imaging depth of the OCT system and $s$ is the ratio between the spectral resolution to the sampling interval. The contribution of the confocal point spread function and the sensitivity roll-off were determined by fitting the equation for $A(z)$ to the OCT amplitude of a sample with a low concentration of Intralipid $(0.003 \%)$ using four different catheters, for which negligible scattering is assumed, and $Z_{R 0}$ and $s$ were the free running parameters. ${ }^{38,39}$ The median values for $Z_{R 0}$ and $s$ are $0.73 \mathrm{~mm}$ and 1.4 , respectively, which corresponds to reported values. ${ }^{40,41}$ After fitting, all fits were judged by an experienced observer and nonmatching fits (e.g., because of a cyst in the data) were excluded from the analysis. In this way, only accurate representations of the optical attenuation coefficient were included in the analysis. Finally, all obtained attenuation coefficient values were multiplied by 1.4 (refractive index for tissue) yielding values per $\mathrm{mm} .{ }^{42}$

\section{Disclosures}

None of the authors declares any conflict of interest.

\section{Acknowledgments}

This study was funded by an unrestricted research grant from the Cure for Cancer Foundation.

\section{References}

1. R. L. Siegel, K. D. Miller, and A. Jemal, "Cancer statistics, 2015," CA Cancer J. Clin. 65(1), 5-29 (2015).

2. A. Heidenreich et al., "EAU guidelines on prostate cancer. Part 1: screening, diagnosis, and local treatment with curative intent-update 2013," Eur. Urol. 65(1), 124-137 (2014). 
3. U. Lindner et al., "Focal laser ablation for prostate cancer followed by radical prostatectomy: validation of focal therapy and imaging accuracy," Eur. Urol. 57(6), 1111-1114 (2010).

4. S. Madersbacher et al., "Effect of high-intensity focused ultrasound on human prostate cancer in vivo," Cancer Res. 55(15), 3346-3351 (1995).

5. C. M. Moore, D. Pendse, and M. Emberton, "Photodynamic therapy for prostate cancer: a review of current status and future promise," Nat. Clin. Pract. Urol. 6(1), 18-30 (2009).

6. G. Onik et al., "'Male lumpectomy': focal therapy for prostate cancer using cryoablation," Urology 70(6 Suppl.), S16-S21 (2007).

7. M. J. Resnick et al., "Long-term functional outcomes after treatment for localized prostate cancer," N. Engl. J. Med. 368(5), 436-445 (2013).

8. M. Valerio et al., "The role of focal therapy in the management of localised prostate cancer: a systematic review," Eur. Urol. 66(4), 732-751 (2014).

9. W. van den Bos et al., "The safety and efficacy of irreversible electroporation for the ablation of prostate cancer: a multicentre prospective human in vivo pilot study protocol," BMJ Open 4(10), e006382 (2014).

10. B. G. Muller et al., "Imaging modalities in focal therapy: patient selection, treatment guidance, and follow-up," Curr. Opin. Urol. 24(3), 218-224 (2014)

11. I. Ocak et al., "Dynamic contrast-enhanced MRI of prostate cancer at 3 T: a study of pharmacokinetic parameters," AJR Am. J. Roentgenol. 189(4), W192-W201 (2007).

12. S. White et al., "Prostate cancer: effect of postbiopsy hemorrhage on interpretation of MR images," Radiology 195(2), 385-390 (1995).

13. G. J. Tearney et al., "Optical biopsy in human urologic tissue using optical coherence tomography," J. Urol. 157(5), 1915-1919 (1997).

14. B. G. Muller et al., "Prostate cancer diagnosis: the feasibility of needlebased optical coherence tomography," J. Med. Imaging 2(3), 037501 (2015).

15. P. G. Wagstaff et al., "In vivo, percutaneous, needle based, optical coherence tomography of renal masses," J. Vis. Exp. (97), e52574 (2015).

16. B. G. Muller et al., "Prostate cancer diagnosis by optical coherence tomography: first results from a needle based optical platform for tissue sampling," J. Biophotonics 9(5), 490-498 (2016).

17. K. Barwari et al., "Differentiation between normal renal tissue and renal tumours using functional optical coherence tomography: a phase I in vivo human study," BJU Int. 110(8 Pt B), E415-E420 (2012).

18. M. T. Bus et al., "Volumetric in vivo visualization of upper urinary tract tumors using optical coherence tomography: a pilot study," J. Urol. 190(6), 2236-2242 (2013).

19. R. Wessels et al., "The value of optical coherence tomography in determining surgical margins in squamous cell carcinoma of the vulva: a single-center prospective study," Int. J. Gynecol. Cancer 25(1), 112118 (2015).

20. P. McCulloch et al., "No surgical innovation without evaluation: the IDEAL recommendations," Lancet 374(9695), 1105-1112 (2009).

21. P. M. Bossuyt and J. B. Reitsma, and Standards for Reporting of Diagnostic, "The STARD initiative," Lancet 361(9351), 71 (2003).

22. H. S. Cho et al., "High frame-rate intravascular optical frequencydomain imaging in vivo," Biomed. Opt. Express 5(1), 223-232 (2014).

23. F. Prati et al., "Intracoronary optical coherence tomography, basic theory and image acquisition techniques," Int. J. Cardiovasc. Imaging 27(2), 251-258 (2011).

24. H. G. Bezerra et al., "Intracoronary optical coherence tomography: a comprehensive review clinical and research applications," JACC Cardiovasc. Interventions 2(11), 1035-1046 (2009).
25. B. G. Muller et al., "Customized tool for the validation of optical coherence tomography in differentiation of prostate cancer," Technol. Cancer Res. Treat. 16(1), 57-65 (2016).

26. M. Almasian et al., "Validation of quantitative attenuation and backscattering coefficient measurements by optical coherence tomography in the concentration-dependent and multiple scattering regime," J. Biomed. Opt. 20(12), 121314 (2015).

27. G. Gaziev et al., "Defining the learning curve for multiparametric magnetic resonance imaging (MRI) of the prostate using MRI-transrectal ultrasonography (TRUS) fusion-guided transperineal prostate biopsies as a validation tool," BJU Int. 117(1), 80-86 (2016).

28. K. C. Latchamsetty et al., "Experience improves staging accuracy of endorectal magnetic resonance imaging in prostate cancer: what is the learning curve?" Can. J. Urol. 14(1), 3429-3434 (2007).

29. J. K. McKenney, "The present and future of prostate cancer histopathology," Curr. Opin. Urol. 27(5), 464-468 (2017).

30. J. T. Kwak and S. M. Hewitt, "Multiview boosting digital pathology analysis of prostate cancer," Comput. Methods Programs Biomed. 142, 91-99 (2017).

31. A. J. Schaumberg et al., "DeepScope: nonintrusive whole slide saliency annotation and prediction from pathologists at the microscope," Lect. Notes Comput. Sci. 10477, 42-58 (2016).

32. A. V. D'Amico et al., "Optical coherence tomography as a method for identifying benign and malignant microscopic structures in the prostate gland," Urology 55(5), 783-787 (2000).

33. S. Chitchian et al., "Combined image-processing algorithms for improved optical coherence tomography of prostate nerves," J. Biomed. Opt. 15(4), 046014 (2010).

34. S. Chitchian, T. P. Weldon, and N. M. Fried, "Segmentation of optical coherence tomography images for differentiation of the cavernous nerves from the prostate gland," J. Biomed. Opt. 14(4), 044033 (2009).

35. P. P. Dangle et al., "The use of high resolution optical coherence tomography to evaluate robotic radical prostatectomy specimens," Int. Braz. J. Urol. 35(3), 344-353 (2009).

36. J. Lopater et al., "Real-time cancer diagnosis during prostate biopsy: ex vivo evaluation of full-field optical coherence tomography (FFOCT) imaging on biopsy cores," World J. Urol. 34(2), 237-243 (2016).

37. T. A. Ozkan et al., "Interobserver variability in Gleason histological grading of prostate cancer," Scand. J. Urol. 50(6), 420-424 (2016).

38. N. A. Nassif et al., "In vivo high-resolution video-rate spectral-domain optical coherence tomography of the human retina and optic nerve," Opt. Express 12(3), 367-376 (2004).

39. T. G. van Leeuwen, D. J. Faber, and M. C. Aalders, "Measurement of the axial point spread function in scattering media using single-mode fiber-based optical coherence tomography," IEEE J. Sel. Top. Quantum Electron. 9(2), 227-233 (2003).

40. G. van Soest et al., "Atherosclerotic tissue characterization in vivo by optical coherence tomography attenuation imaging," J. Biomed. Opt. 15(1), 011105 (2010).

41. S. Yun et al., "High-speed optical frequency-domain imaging," Opt. Express 11(22), 2953-2963 (2003).

42. D. M. de Bruin et al., "Optical phantoms of varying geometry based on thin building blocks with controlled optical properties," J. Biomed. Opt. 15(2), 025001 (2010).

Biographies for the authors are not available. 